\title{
A Mathematical Model to Investigate the Long-Term Effects of the Lymphatic Filariasis Medical Treatment in Jati Sampurna, West Java
}

\author{
A.K. Supriatna ${ }^{1, *}$, H. Serviana ${ }^{2}$ \& E. Soewono ${ }^{3}$ \\ ${ }^{1}$ Department of Mathematics, Universitas Padjadjaran Bandung \\ ${ }^{2}$ Department of Mathematics, Universitas Islam Negeri Makassar \\ ${ }^{3}$ Industrial and Financial Mathematics Group, Institut Teknologi Bandung \\ * Corresponding author: aksupriatna@bdg.centrin.net.id
}

\begin{abstract}
In this paper we discuss a mathematical model for the transmission of Lymphatic Filariasis disease in Jati Sampurna, West Java Indonesia. The model assumes that acute infected humans are infectious and treatment is given to a certain number of acute infected humans found from screening process. The treated acute individuals are assumed to be remain susceptible to the disease. The model is analyzed and it is found a condition for the existence and stability of the endemic equilibrium. A well known rule of thumb in epidemiological model, that is, the endemic equilibrium exists and stable if the basic reproduction number is greater than one, is shown. Moreover, it is also shown that if the level of screening $n$ is sufficiently large, current medical treatment strategy will be able to reduce the long-term level of incidences. However, in practice it is not realistic and cannot eliminate the disease, in terms of reducing the basic reproduction number. The reproduction number can be reduced by giving additional treatments, such as reducing the biting rate and mosquito's density. This suggests that there should be a combination of treatment to eliminate the disease.
\end{abstract}

Keywords: Filariasis; mathematical model; basic reproduction number.

\section{$1 \quad$ Introduction}

Elephantiasis, which is often known as lymphatic filariasis, is regarded as a parasitic dangerous disease. It may cause a chronic morbidity if the persons who are infected are left untreated. Even successful treatment may still left the persons in a cripple condition for life that makes psychological and economical burden, both for them and for the society in general. The disease is characterized by the thickening of the skin and underlying tissues. Most of the cases are in the legs and genital areas, as results from the damages of the lymphatic vessels. The disease is mainly caused by Wuchereria bancrofti parasites and transmitted by Culex spp. mosquitoes. 
Due to the widespread of the incidences, especially in many tropical countries, a global program to eliminate lymphatic filariasis was initiated in 1998 to target the worldwide elimination of this parasitic disease [1]. Among the strategies are transmission interruption and morbidity management. Transmission interruption was done by treating infected population with anti-filarial drugs. Morbidity management was done to reduce the suffering of patients who have acute and chronic manifestations. In 2004 alone there were more than thirty countries have started elimination program and this number is still escalating [2].

Various degrees of success have emerged as a result of the implementation of this program. Although it was reported that in some places the program has interrupted the transmission, but in many other places the program could not stop the transmission of the disease [2]. It has been argued that strategic choices and operational or biological factors contribute to the success or failure of the program [2]. However, sometimes it is difficult to evaluate the success or the failure of a health program, especially in the beginning of the program.

Since in many cases a mathematical model has provided useful tools for planning and evaluation of control program in disease elimination [3], in this paper we discuss, via mathematical modeling, a program set up by the Indonesian government in the effort to eliminate the disease. We use a set of equations as a model of the disease transmission and analyze the model to help us to explore and understand the complex transmission dynamics of this parasitic disease, from which some insight and interpretation could be derived. The model discussed here is developed from a local context by looking at current filariasis treatment in Jati Sampurna Bekasi, West Java.

In general, mathematical models for the transmission of disease caused by parasites, such as filariasis, at least fall into two different categories. In the first category are those that deal with only the process of infection, in the sense of the presence and absence of the disease in the host caused by the parasites. While in the second category, are those deal not merely in the presence and absence of parasites, but also with the count of the parasistes burden in an individual host. Among the example in the first category is [4]. While [5] and [6] are examples of the second category for general parasite infections, and [7] and $[8,9]$ are examples for the case of filariasis infection. Since filariasis is transmitted via mosquitoes, $[10,11]$ also modeled the filariae count in the vector. The author in [12] discussed different categorization.

Although mathematical models for the transmission and controlling filariasis are abundance, however, there is no model related to the current treatment of filariasis in Jatisampurna, Indonesia. In this paper we explore the effectiveness of this filariasis treatment by developing a model that considers a host-vector 
transmission. Although parasite burden is important, especially in determining the degree of infection, for our study we limit to the presence and absence of infection in host and vector individuals. We follow a standar SI model both for the host and the vector with and addition of treatment. We show that although current treatment can reduce the number of filarial prevalence, but in the long run it will not able to eliminate the disease completely, unless different strategy is used. This is partially due to the fact that host individuals successfully treated may still reinfected.

The incidence of filariasis in Jati Sampurna is regarded as the highest in Indonesia [13]. For this reason, the Indonesian Government has set up an intensive program to eliminate the disease in that region. The current practice of the treatment in the region can be described briefly as follow. For every new single chronic found, a health worker undertakes screening to test $n$ number of nearby individuals. If an infected individual is found in this screening test, treatment is given to this individual [14]. This strategy implicitly based on an assumption that the chronic is no longer infectious, for example due to isolation from the rest of the population. We devise a mathematical model to investigate the long-run effects of this existing strategy in the transmission of the disease.

\section{$2 \quad$ Mathematical Model}

To formulate the mathematical model we use assumptions that initially the human population is virgin, the total population of human is constant, there is only one species of worm and one species of mosquito, and there is no vertical transmission of the disease. The human population is divided into three subpopulations, susceptible $S_{h}$, infected-carrier $A$ and infected-chronic $K$ populations, with total number $N_{h}$. The mosquitoes is divided in two subpopulation, susceptible $S_{V}$ and infected $I_{V}$ mosquitoes, with total number $N_{V}$. Related parameters in the model are the human recruitment rate $R_{h}$, human death rate $\mu_{h}$, successful rate of transmission from mosquitoes to susceptible human $p_{h}$, mosquitoes biting rate on human $b$, symptomatic rate $\delta$, mosquitoes recruitment rate $R_{V}$, mosquitoes death rate $\mu_{V}$ and successful rate of filaria transmission from human to susceptible mosquitos $p_{V}$. If the medical treatment is quantified by the number of people screened by the health authority $n$, with the probability of successful treatment $p_{0}$, then the governing differential equations as the mathematical model of the disease transmission are 


$$
\begin{aligned}
& S_{h}^{\prime}(t)=R_{h}-\frac{b I_{v} S_{h} p_{h}}{N_{h}}-\mu_{h} S_{h}+\frac{p_{o} n A^{2} \delta}{N_{h}} \\
& A^{\prime}(t)=\frac{b I_{v} S_{h} p_{h}}{N_{h}}-\delta A-\mu_{h} A-\frac{p_{o} n A^{2} \delta}{N_{h}} \\
& K^{\prime}(t)=\delta A-\mu_{h} K \\
& S_{v}^{\prime}(t)=R_{v}-\frac{b S_{v} A p_{v}}{N_{h}}-\mu_{v} S_{v} \\
& I_{v}^{\prime}(t)=\frac{b S_{v} A p_{v}}{N_{h}}-\mu_{v} I_{v}
\end{aligned}
$$

The next sections discuss the endemic and non-endemic steady states of the model related to the basic reproduction ratio to evaluate the effectiveness of the medical treatment $n$ of the current policy in managing the disease.

\section{Endemic and Non-endemic Steady States}

To analyze the model we assume that the host and vector populations are constant, so that $N_{h}=\frac{R_{h}}{\mu_{h}}$ and $N_{v}=\frac{R_{v}}{\mu_{v}}$. We will derive the basic reproduction number via the next generation matrix [15]. We will show that the existence and the stability of the endemic equilibrium depend on the basic reproduction number $R_{0}$. We will also show that the number of acute population is inversely proportional to the level of screening $n$.

In the steady state condition, the densities of the vector are given by

$$
S_{v}^{*}=\frac{R_{v}}{b \frac{A}{N_{h}} p_{v}+\mu_{v}}
$$

and

$$
I_{v}^{*}=\frac{R_{v} b A p_{v}}{\mu_{v} N_{h}\left(b \frac{A}{N_{h}} p_{v}+\mu_{v}\right)} .
$$

Substituting these vector densities into (1)-(2) yields a reduced form of the transmission model 


$$
\begin{aligned}
& S_{h}^{\prime}(t)=R_{h}-f\left(S_{h}, A\right)-\mu_{h} S_{h}+\frac{p_{o} n A^{2} \delta}{N_{h}}, \\
& A^{\prime}(t)=f\left(S_{h}, A\right)-\left(\delta+\mu_{h}\right) A-\frac{p_{o} n A^{2} \delta}{N_{h}}, \\
& K^{\prime}(t)=\delta A-R_{h}-\mu_{h} S_{h}-\mu_{h} A,
\end{aligned}
$$

where

$$
f\left(S_{h}, A\right)=\frac{b^{2} R_{v} A S_{h} p_{v} p_{h} \mu_{h}^{2}}{\mu_{v} R_{h}\left(b A \mu_{h} p_{v}+\mu_{v} R_{h}\right)}
$$

is a nonlinear incidence rate satisfying

$$
\begin{aligned}
& f(0, A)=f\left(S_{h}, 0\right)=0, \\
& \frac{\partial f\left(S_{h}, A\right)}{\partial S_{h}}>0, \frac{\partial f\left(S_{h}, A\right)}{\partial A}>0,
\end{aligned}
$$

and

$$
\frac{\partial^{2} f\left(S_{h}, A\right)}{\partial A^{2}} \leq 0 .
$$

The basic reproduction number can be obtained using the next generation matrix from the reduced model, equations (8)-(10), with the resulting expression given by

$$
R_{0}=\frac{\sqrt{b^{2} R_{h} R_{v} \mu_{h} p_{v} p_{h}\left(\delta+\mu_{h}\right)}}{R_{h} \mu_{v}\left(\delta+\mu_{h}\right)} .
$$

It is worth to note that the basic reproduction number does not depend on the level of screening $n$, and hence, current method of treatment does not annihilate the endemicity of the disease. This is partially because of the resusceptibility of the treated population. However, we will show that it indeed reduces the number of the acute population in the long-term.

From equation (9), the non-trivial steady state acute population satisfies

$$
c_{1} A^{2}+c_{2} A+c_{3}=0,
$$

where

$$
\begin{aligned}
& c_{1}=\mu_{v} b \mu_{h}^{2} p_{v} p_{o} n \delta, \\
& c_{2}=\mu_{v} R_{h} b \mu_{h} p_{v}\left(\delta+\mu_{h}\right)+\mu_{v}^{2} R_{h} \alpha n \mu_{h} \delta,
\end{aligned}
$$


$c_{3}=\mu_{v}^{2} R_{h}^{2}\left(\delta+\mu_{h}\right)-S_{h} b^{2} R_{v} p_{v} p_{h} \mu_{h}^{2}$.

while from equation (8), the susceptible population satisfies

$$
S_{h}=\frac{R_{h}-A\left(\delta+\mu_{h}\right)}{\mu_{h}} .
$$

Substituting the last equation into (16) gives

$$
\omega n A^{2}+\rho A+\alpha n A+\theta=0,
$$

where

$$
\begin{aligned}
\omega & =\mu_{v} b \mu_{h}^{2} p_{v} p_{o} \delta \\
\rho & =\mu_{v} R_{h} b \mu_{h} p_{v}\left(\delta+\mu_{h}\right)+b^{2} R_{v}\left(\delta+\mu_{h}\right)+p_{v} p_{h} \mu_{h} \\
\alpha & =\mu_{v}^{2} R_{h} \alpha \mu_{h} \delta \\
\theta & =\mu_{v}^{2} R_{h}^{2}\left(\delta+\mu_{h}\right)-R_{h} b^{2} R_{v} p_{v} p_{h} \mu_{h} .
\end{aligned}
$$

We see from the last equation that $A$ exists if and only if $\theta<0$, or equivalently, $\bar{R}>1$, where

$$
\bar{R}=\frac{b^{2} R_{v} p_{v} \mu_{h} p_{h}}{R_{h} \mu_{v}^{2}\left(\delta+\mu_{h}\right)}
$$

Note that $\bar{R}=R_{0}^{2}$. Hence, we conclude that the non-trivial equilibrium acute population exists if and only if the basic reproduction number $R_{0}$ is greater than one. Furthermore we note that equation (18) is equivalent to

$$
n=-\left(\frac{\theta}{A}+\rho\right)\left(\frac{1}{\omega A+A}\right) \text { with } \theta<0 .
$$

This implies that the long-term acute population density $A^{*}$ decreases whenever $n$ increases.

To analyze the stability of the endemic-free steady state $E_{1}=\left(S_{h 0}^{*}, A_{0}^{*}\right)=\left(\frac{R_{h}}{\mu_{h}}, 0\right)$ and the endemic steady state $E_{2}=\left(S_{h 1}^{*}, A_{1}^{*}\right)$ we proceed by proving the following Lemmas. 
Lemma 1 At the endemic-free equilibrium state $E_{1}=\left(S_{h 0}^{*}, A_{0}^{*}\right)=\left(\frac{R_{h}}{\mu_{h}}, 0\right)$, the following properties hold

$$
\begin{aligned}
& R_{0} \leq 1 \Rightarrow \frac{\partial f\left(S_{h 0}^{*}, A_{0}^{*}\right)}{\partial A} \leq \delta+\mu_{h}, \\
& R_{0}>1 \Rightarrow \frac{\partial f\left(S_{h 0}^{*}, A_{0}^{*}\right)}{\partial A}>\delta+\mu_{h} .
\end{aligned}
$$

Proof. Note that there is a relationship between the incidence rate (11) and the basic reproduction number (15) in the form

$$
R_{0}^{2}=\bar{R}=\frac{b^{2} R_{v} p_{v} \mu_{h} p_{h}}{R_{h} \mu_{v}^{2}\left(\delta+\mu_{h}\right)}=\left.\frac{1}{\delta+\mu_{h}} \frac{\partial f\left(S_{h}, A\right)}{\partial A}\right|_{\left(S_{h}, A_{h}\right)=\left(S_{h 0}^{*}, A_{0}^{*}\right)}
$$

Clearly, if $\quad R_{0} \leq 1 \quad$ then $\quad \frac{1}{\delta+\mu_{h}} \frac{\partial f\left(S_{h 0}^{*}, A_{0}^{*}\right)}{\partial A} \leq 1 \quad$ or $\quad$ equivalently $\frac{\partial f\left(S_{h 0}^{*}, A_{0}^{*}\right)}{\partial A} \leq \delta+\mu_{h}$. The second part of the lemma can be proved similarly.

Lemma 2 At the endemic equilibrium state $E_{2}=\left(S_{h 1}^{*}, A_{1}^{*}\right)$, the following properties hold

$$
R_{0}>1 \Rightarrow \frac{\partial f\left(S_{h 1}^{*}, A_{1}^{*}\right)}{\partial A} \leq \delta+\mu_{h}+2 p_{o} n \frac{A_{1}^{*}}{R_{h}} \mu_{h} \delta .
$$

Proof. We observe, from (9), that the endemic equilibrium state satisfies

$$
f\left(S_{h 1}^{*}, A_{1}^{*}\right)=\left(\delta+\mu_{h}\right) A_{1}^{*}+p_{o} n \frac{\left(A_{1}^{*}\right)^{2}}{R_{h}} \mu_{h} \delta .
$$

Define the following function,

$$
g\left(A_{h 1}^{*}\right)=f\left(S_{h 1}^{*}, A_{1}^{*}\right) .
$$

Suppose that, in the contrary, we have

$$
\frac{\partial f\left(S_{h 1}^{*}, A_{1}^{*}\right)}{\partial A}=\frac{d g\left(A_{1}^{*}\right)}{d A}>\delta+\mu_{h}+2 p_{o} n \frac{A_{1}^{*}}{R_{h}} \mu_{h} \delta .
$$


By considering (12), the mean value theorem guarantees that there is a point $A_{2} \in\left(0, A_{1}^{*}\right)$ such that

$$
\frac{d g\left(A_{2}\right)}{d A}=\frac{g\left(A_{1}^{*}\right)-g(0)}{A_{1}^{*}-0}=\delta+\mu_{h}+p_{o} n \frac{A_{1}^{*}}{R_{h}} \mu_{h} \delta .
$$

Moreover since we assumed $\frac{d g\left(A_{1}^{*}\right)}{d A}>\delta+\mu_{h}+2 p_{o} n \frac{A_{1}^{*}}{R_{h}} \mu_{h} \delta$, then again by the mean value theorem there exist point $A_{3} \in\left(A_{2}, A_{1}^{*}\right)$ such that

$$
\begin{gathered}
\frac{\partial^{2} f\left(S_{h 1}^{*}, A_{3}\right)}{\partial A^{2}}=\frac{d^{2} g\left(A_{3}\right)}{d A^{2}}=\frac{\frac{d g\left(A_{1}^{*}\right)}{d A}-\frac{d g\left(A_{2}\right)}{d A}}{A_{1}^{*}-A_{2}} \\
=\frac{\frac{d g\left(A_{1}^{*}\right)}{d A}-\left(\delta+\mu_{h}+p_{o} n \frac{A_{1}^{*}}{R_{h}} \mu_{h} \delta\right)}{A_{1}^{*}-A_{2}}>0 .
\end{gathered}
$$

This contradicts (14), and hence $\frac{\partial f\left(S_{h l}^{*}, A_{1}^{*}\right)}{\partial A} \leq \delta+\mu_{h}+2 p_{o} n \frac{A_{1}^{*}}{R_{h}} \mu_{h} \delta$.

Using Lemma 1 it is easy to prove that the endemic-free equilibrium is locally asymptotically stable if $R_{0}<1$. A similar result for the endemic equilibrium is summarized in the following theorem.

Theorem 1 If $R_{0}>1$ then the endemic equilibrium of the system is locally asymptotically stable, otherwise it is unstable.

Proof. The Jacobian matrix of the system is given by

$$
M=\left(\begin{array}{cc}
-\frac{\partial f}{\partial S_{h}}-\mu_{h} & -\frac{\partial f}{\partial A}+\frac{2 p_{o} n A \mu_{h} \delta}{R_{h}} \\
\frac{\partial f}{\partial S_{h}} & \frac{\partial f}{\partial A}-\left(\delta+\mu_{h}+\frac{2 p_{o} n A \mu_{h} \delta}{R_{h}}\right)
\end{array}\right)
$$

in which 


$$
\begin{aligned}
\operatorname{det} M & =\left(-\frac{\partial f}{\partial S_{h}}-\mu_{h}\right)\left(\frac{\partial f}{\partial A}-\left(\delta+\mu_{h}+\frac{2 p_{o} n A \mu_{h} \delta}{R_{h}}\right)\right) \\
- & \frac{\partial f}{\partial S_{h}}\left(-\frac{\partial f}{\partial A}+\frac{2 p_{o} n A \mu_{h} \delta}{R_{h}}\right) .
\end{aligned}
$$

From Lemma 2 we have

$$
\begin{aligned}
& \operatorname{det} M=\mu_{h}\left(\left(\delta+\mu_{h}+\frac{2 p_{o} n A \mu_{h} \delta}{R_{h}}\right)-\frac{\partial f}{\partial A}\right)+\frac{\partial f}{\partial S_{h}}\left(\delta+\mu_{h}\right)>0, \\
& \text { trace } M=-\frac{\partial f}{\partial S_{h}}-\mu_{h}+\frac{\partial f}{\partial A}-\left(\delta+\mu_{h}+\frac{2 p_{o} n A \mu_{h} \delta}{R_{h}}\right)<0 .
\end{aligned}
$$

This proves the theorem.

\section{$4 \quad$ Numerical Simulation}

To illustrate the results presented in the previous sections, we give a numerical example representing the case in Jati Sampurna (West Java Indonesia) and use the parameters: $p_{h}=0.001, \mu_{h}=\frac{1}{65}$ per year, $\mu_{v}=12.67$ per year, $p_{v}=0.001$, $p_{0}=0.9$ per year, $b=243$ per capita, $\delta=0.2$ per year. We assume that there is one acute individual in a nearly virgin population. There is no chronic individual and no infected mosquito initially.

The upper part of Figure 1 shows that if there is no medical treatment, $n=0$, the peaks for the acute and chronic populations are at the level of 7,432 and 13,275 individuals, respectively. These peaks are attained 14.6 and 30.3 years after the introduction of the first human infection, respectively. In the long-term the equilibrium levels for the acute and chronic populations are 906 and 11,784, which shows a severe endemicity of the disease. The lower part of Figure 1 shows that if there is a medical treatment with $n=100$, the peaks for the acute and chronic populations are much lower than that for $n=0$ in Figure 1. In the long-term the equilibrium levels for the acute and chronic populations also decrease, which shows that the level of the endemicity of the disease reduces. 

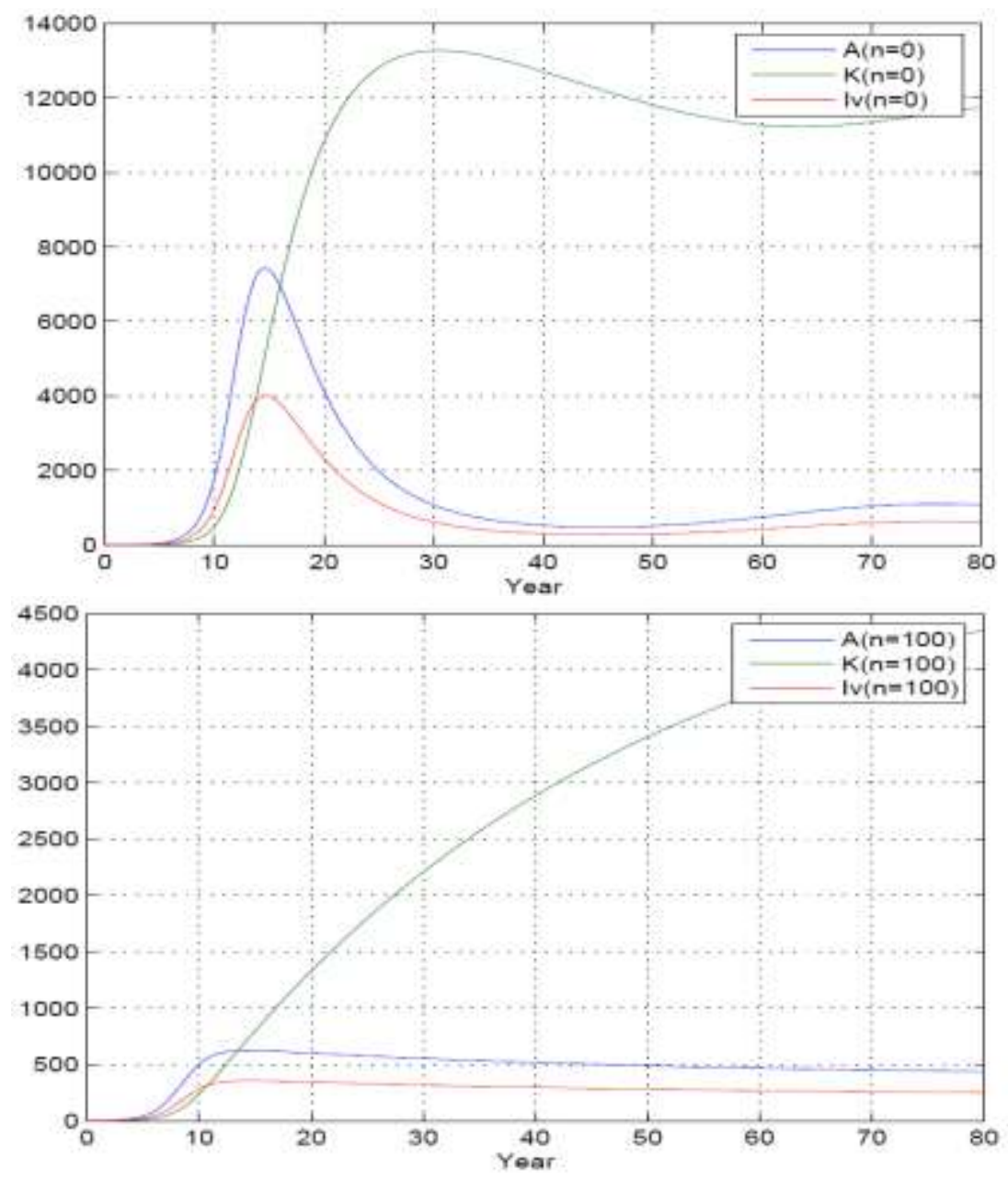

Figure 1 The upper figure shows that if there is no medical treatment, $n=0$, the peaks for the acute and chronic populations are at the level of 7,432 and 13,275 individuals, respectively. These peaks are attained 14.6 and 30.3 years after the introduction of the first human infection,respectively. In the long-term the equilibrium levels for the acute and chronic populations are 906 and 11,784, which shows a severe endemicity of the disease. The lower figure shows that if there is a medical treatment with $n=100$, the peaks for the acute and chronic populations are lower than that for $n=0$ in the left figure. In the long-term the equilibrium levels for the acute and chronic populations also decrease, which shows that the level of the endemicity of the disease reduces. 

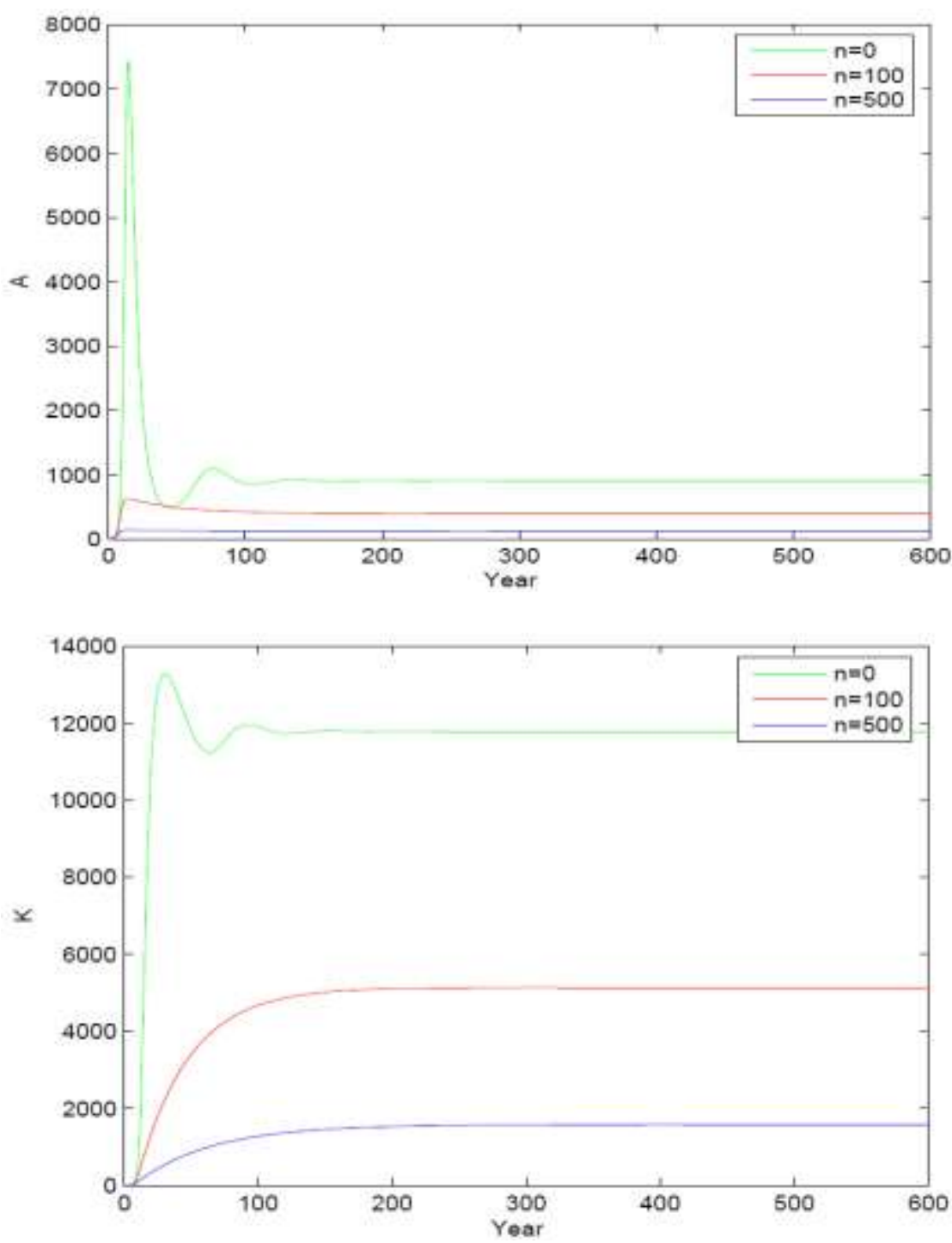

Figure 2 The figure show the reduction of the endemicity of the Acute (upper) and chronic (lower) infected human populations as the level of screening $n$ varies.

Figures 2 shows the reduction of the endemicity as the level of screening $n$ varies. The last figure (Figure 3) shows the reduction of $R_{0}$ as the biting rate, vector density, and the ratio of vector and human densities reduce to a certain percentage of existing level. This figure helps to identify the effort needed to reduce the basic reproduction number in order to prevent the transmission of the 
disease. It shows that reducing the biting rate is more effective than reducing the vector density. This easy to understand since the biting rate term appears as a quadratic form in the $R_{0}$ expression. For example, reducing the biting rate to $40 \%$ from existing level will result in the reduction of $R_{0}$ from nearly 5.5 to just above 3 , while reducing the vector density to $40 \%$ from existing level will only result in the reduction of $R_{0}$ to approximately 4 .

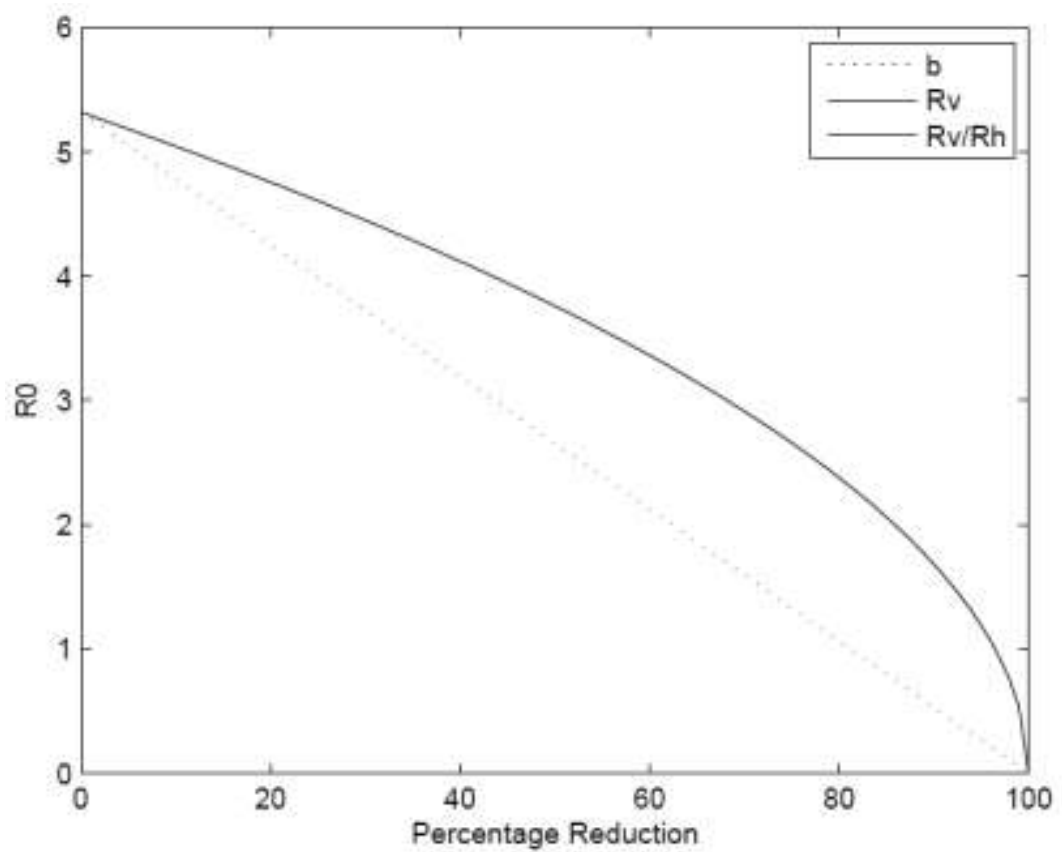

Figure 3 The reduction of $R_{0}$ as the biting rate (dots) and vector density (solid) reduce to a certain percentage of existing level. For example, reducing the biting rate to $40 \%$ from existing level will result in the reduction of $R_{0}$ from nearly 5.5 to approximately just above 3 . The curve of vector density reduction $\left(R_{V}\right)$ coincides with the curve of vector per human index reduction $\left(R_{V} / R_{H}\right)$.

\section{Acknowledgement}

Financial support was provided by the Royal Netherlands Academy of Arts and Sciences (KNAW) through the SPIN Postdoc Project. The work was partly funded by ITB Research Grant 036/K01.7/PL/2008 to the third author. 


\section{References}

[1] Molyneux, D., Zagaria, N., Lymphatic Filariasis Elimination: Progress In Global Programme Development, Ann. Trop. Med. Parasitol, 96 (Suppl 2): S15-40, 2002.

[2] World Health Organization, Global Programme To Eliminate Lymphatic Filariasis-Progress Report For 2004, Wkly. Epidemiol. Rec., 80, pp. 202-212, 2005.

[3] Goodman, B., Models Aid Understanding, Help Control Parasites [News], Science, 264, pp. 1862-1863, 1994.

[4] Li, G., Wang, W., Wang, K. \& Jin, Z., Dynamic Behavior Of A ParasiteHost Model With General Incidence, J. Math. Anal. and Appl., 331, pp. 631-643, 2007.

[5] Hadeler, K.P. \& Dietz, K., Nonlinear Hyperbolic Partial Differential Equations For The Dynamics of Parasite Populations, Computer and Mathematics with Application, 9(3) 415-430, 1983.

[6] Milner, F.A. \& Patton, C.A., A New Approach to Mathematical Modeling of Host-Parasite Systems, Comp. and Math. with Appl, 37, pp. 93-110, 1999.

[7] Chan, M.S., Srividya, A., Norman, R.A., Pani, S.P., Ramaiah, K.D., Vanamail, P., Michael, E., Das, P.K. \& Bundy, D.A., Epifil: A Dynamicmodel Of Infection And Disease In Lymphatic Filariasis, Am. J. Trop. Med. Hyg, 59, pp. 606-614, 1998.

[8] Michael, E., Malecela-Lazaro, M.N., Kabali, C., Snow, L.C. \& Kazura, J.W., Mathematical Models And Lymphatic Filariasis Control: Endpoints And Optimal Interventions Edwin, Trends In Parasitol, 22(5), 2006.

[9] Michael, E., Malecela-Lazaro, M.N., Maegga, B., Fischer, P. \& Kazura, J.W., Mathematical Models And Lymphatic Filariasis Control: Monitoring And Evaluating Interventions, Trends In Parasitol, 22(11), 2006.

[10] Snow, L.C. \& Michael, E., Transmission Dynamic Of Limpatic Filariasis: Density Dependent In The Uptake Of Wucheria Bancrofti Microfilariae By Vector Mosquitoes, Medical And Veterinary Entomology, 16, pp. 409-423, 2002.

[11] Stolk, et al., Assessing Density Dependence In The Transmission Of Lymphatic Filariasis: Uptake And Development of wuchereria Bancrofti Microfilariae in the Vectormosquitoes, Medical And Veterinary Entomology, 18, pp. 57-60, 2004.

[12] Roberts, M., A Pocket Guide To Host-Parasite Models, Parasitol. Today 11(5), pp. 172-177, 1995.

[13] Anonim, Annual Report of Filariasis Patient in Jati Sampurna, Jati Sampurna, Indonesia, 2005. 
[14] Dirjen PPPL, Pedoman Penentuan dan Evaluasi Daerah Endemis Filariasis, Health Department of the Republic of Indonesia, Jakarta, 2006.

[15] Diekmann, O. \& Heesterbeek, J.A.P., Mathematical Epidemiology of Infectious Diseases, John Wiley \& Son, New York, 2000. 